\title{
Water abundance variations around high-mass protostars: HIFI observations of the DR21 region $\star$
}

\author{
F. F. S. van der Tak ${ }^{1,2}$, M. G. Marseille ${ }^{1}$, F. Herpin ${ }^{3}$, F. Wyrowski ${ }^{4}$, A. Baudry ${ }^{3}$, S. Bontemps ${ }^{3}$, J. Braine ${ }^{3}$, S. Doty ${ }^{13}$, \\ W. Frieswijk ${ }^{2}$, G. Melnick ${ }^{12}$, R. Shipman ${ }^{1}$, E. F. van Dishoeck ${ }^{5,14}$, A. O. Benz ${ }^{9}$, P. Caselli ${ }^{15}$, M. Hogerheijde ${ }^{5}$, \\ D. Johnstone ${ }^{16,17}$, R. Liseau ${ }^{8}$, R. Bachiller ${ }^{18}$, M. Benedettini ${ }^{19}$, E. Bergin ${ }^{20}$, P. Bjerkeli ${ }^{8}$, G. Blake ${ }^{21}$, S. Bruderer ${ }^{9}$, \\ J. Cernicharo ${ }^{22}$, C. Codella ${ }^{19}$, F. Daniel ${ }^{31,22}$, A. M. di Giorgio ${ }^{19}$, C. Dominik ${ }^{23}$, P. Encrenaz ${ }^{24}$, M. Fich ${ }^{25}$, A. Fuente ${ }^{18}$, \\ T. Giannini ${ }^{19}$, J. Goicoechea ${ }^{22}$, Th. de Graauw ${ }^{10}$, F. Helmich ${ }^{1}$, G. Herczeg ${ }^{14}$, J. Jørgensen ${ }^{26}$, L. Kristensen ${ }^{5}$, \\ B. Larsson ${ }^{27}$, D. Lis ${ }^{21}$, C. McCoey ${ }^{25}$, D. Neufeld ${ }^{28}$, B. Nisini ${ }^{19}$, M. Olberg ${ }^{8}$, B. Parise ${ }^{4,6}$, J. Pearson ${ }^{29}$, R. Plume ${ }^{30}$, \\ C. Risacher ${ }^{1}$, J. Santiago ${ }^{18}$, P. Saraceno ${ }^{19}$, M. Tafalla ${ }^{18}$, T. van Kempen ${ }^{12}$, R. Visser ${ }^{5}$, S. Wampfler ${ }^{9}$, U. Y 1 Idiz $^{5}$, \\ L. Ravera ${ }^{7}$, P. Roelfsema ${ }^{1}$, O. Siebertz ${ }^{6}$, and D. Teyssier ${ }^{11}$
}

(Affiliations are available in the online edition)

Received 26 March 2010 / Accepted 20 April 2010

\section{ABSTRACT}

Context. Water is a key molecule in the star formation process, but its spatial distribution in star-forming regions is not well known.

Aims. We study the distribution of dust continuum and $\mathrm{H}_{2} \mathrm{O}$ and ${ }^{13} \mathrm{CO}$ line emission in DR21, a luminous star-forming region with a powerful outflow and a compact H II region.

Methods. Herschel-HIFI spectra near $1100 \mathrm{GHz}$ show narrow ${ }^{13} \mathrm{CO} 10-9$ emission and $\mathrm{H}_{2} \mathrm{O}_{11}-0_{00}$ absorption from the dense core and broad emission from the outflow in both lines. The $\mathrm{H}_{2} \mathrm{O}$ line also shows absorption by a foreground cloud known from ground-based observations of low- $J$ CO lines.

Results. The dust continuum emission is extended over 36 " FWHM, while the ${ }^{13} \mathrm{CO}$ and $\mathrm{H}_{2} \mathrm{O}$ lines are confined to $\approx 24^{\prime \prime}$ or less. The foreground absorption appears to peak further North than the other components. Radiative transfer models indicate very low abundances of $\sim 2 \times 10^{-10}$ for $\mathrm{H}_{2} \mathrm{O}$ and $\sim 8 \times 10^{-7}$ for ${ }^{13} \mathrm{CO}$ in the dense core, and higher $\mathrm{H}_{2} \mathrm{O}$ abundances of $\sim 4 \times 10^{-9}$ in the foreground cloud and $\sim 7 \times 10^{-7}$ in the outflow. Conclusions. The high $\mathrm{H}_{2} \mathrm{O}$ abundance in the warm outflow is probably due to the evaporation of water-rich icy grain mantles, while the $\mathrm{H}_{2} \mathrm{O}$ abundance is kept down by freeze-out in the dense core and by photodissociation in the foreground cloud.

Key words. ISM: molecules - stars: formation - astrochemistry - ISM: individual objects: DR21

\section{Introduction}

The water molecule is a key species throughout the formation of stars and planets. In the gas phase, it acts as a coolant of collapsing interstellar clouds; in the solid state, it acts as glue for dust grains in protoplanetary disks to make planetesimals; and as a liquid, it acts as transporter bringing molecules together on planetary surfaces, a key step towards biogenic activity. The first role is especially important for high-mass star formation which depends on the balance between the collapse of a massive gas cloud and its fragmentation (Zinnecker \& Yorke 2007).

Interstellar $\mathrm{H}_{2} \mathrm{O}$ is well known from ground-based observations of the $22 \mathrm{GHz}$ maser line. Previous space-based submm and far-IR observations have measured $\mathrm{H}_{2} \mathrm{O}$ abundances ranging from $10^{-8}$ in cold gas to $10^{-4}$ in warm gas (ISO: Van Dishoeck \& Helmich 1996; SWAS: Melnick \& Bergin 2005; Odin: Bjerkeli et al. 2009) but did not have sufficient angular resolution to determine the spatial distribution of $\mathrm{H}_{2} \mathrm{O}$. In contrast, space-based mid-IR and ground-based mm-wave observations have high

* Herschel is an ESA space observatory with science instruments provided by European-led Principal Investigator consortia and with important participation from NASA. angular resolution but only probe the small fraction of the gas at high temperatures (Van der Tak et al. 2006; Watson et al. 2007).

This paper presents observations of an $\mathrm{H}_{2} \mathrm{O}$ ground state line at $>3 \times$ higher angular resolution than previously possible for such lines. Through radiative transfer models, we compare the abundance distribution of $\mathrm{H}_{2} \mathrm{O}$ with that of ${ }^{13} \mathrm{CO}$ and dust. The source DR21 (Main) is a high-mass protostellar object $\left(L=45000 L_{\odot}\right)$ located in the Cygnus $\mathrm{X}$ region at $d=$ $1.7 \mathrm{kpc}$ (Schneider et al. 2006), about 3' South of the well-known DR21(OH) object (also known as W75S). Maps of the $1.2 \mathrm{~mm}$ dust emission show a dense core with a mass of $600-1000 M_{\odot}$ and a size of $0.19 \times 0.14 \mathrm{pc}$ FWHM, surrounded by an extended envelope with mass $4750 M_{\odot}$ and size $0.3 \mathrm{pc}$ (Motte et al. 2007). Gas densities of $10^{5}-10^{6} \mathrm{~cm}^{-3}$ are derived from both the mm-wave continuum and $\mathrm{HCN}$ and $\mathrm{HCO}^{+}$line emission (Kirby 2009). Signs of active high-mass star formation are the bright mid-IR emission (272 Jy at $21 \mu \mathrm{m}$ ), the presence of an $\mathrm{H}_{2} \mathrm{O}$ $22 \mathrm{GHz}$ maser (see catalog of Braz \& Epchtein 1983) and emission from ionized gas extending over 20-30" (Roelfsema et al. 1989). Together with the powerful molecular outflow (Garden et al. 1991) these signs indicate that the source is relatively evolved within the embedded phase of high-mass star formation, beyond the "ultracompact H II region" phase. 

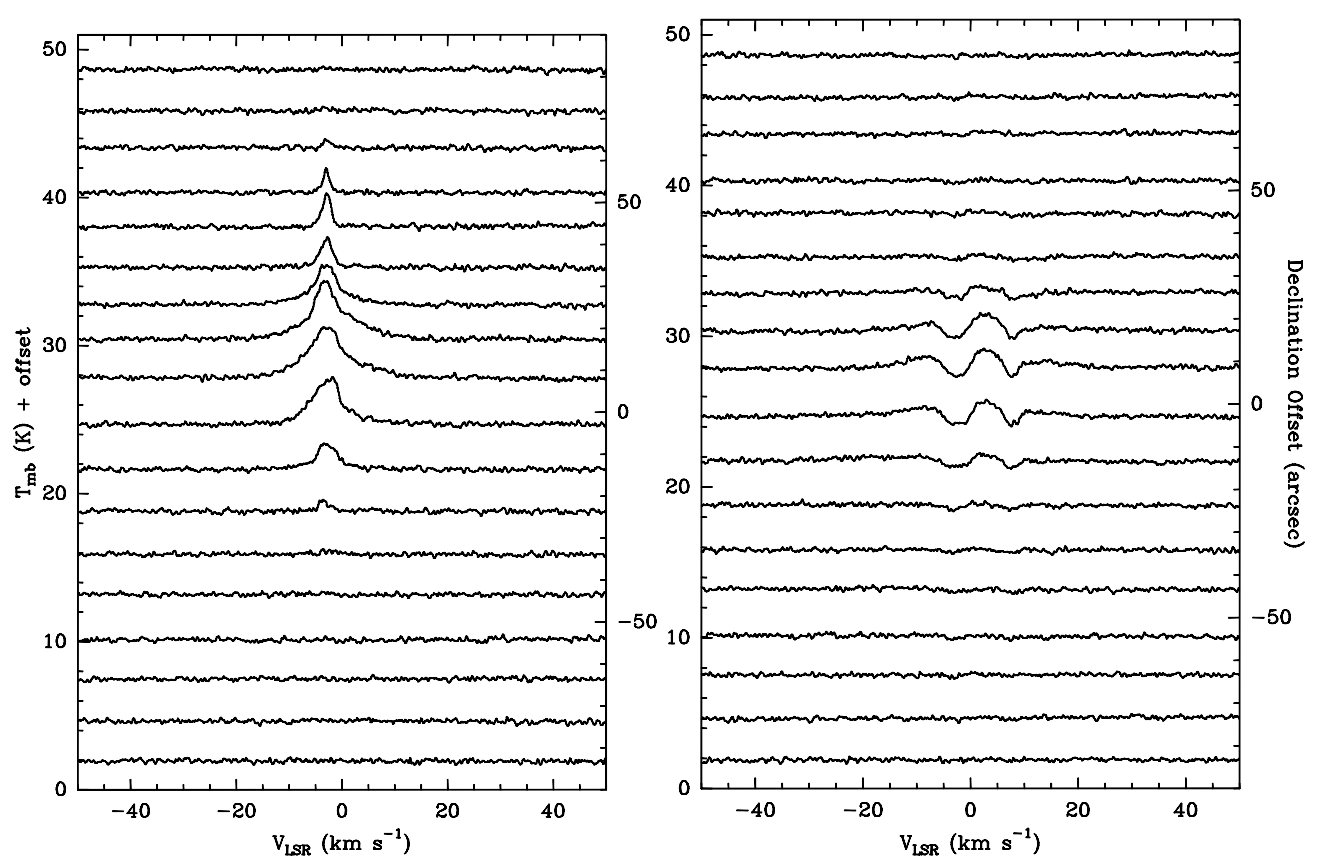

Fig. 1. Spectra of ${ }^{13} \mathrm{CO} 10-9$ (left) and $\mathrm{H}_{2} \mathrm{O} 1_{11}-0_{00}$ (right) lines toward DR21, taken with the WBS backend.

\section{Observations}

The DR21 region was observed with the Heterodyne Instrument for the Far-Infrared (HIFI; de Graauw et al. 2010) onboard ESA's Herschel Space Observatory (Pilbratt et al. 2010) on June 22, 2009. Spectra were taken in double sideband mode using receiver band $4 \mathrm{~b}$, with $v_{\mathrm{LO}}=1107.990 \mathrm{GHz}$ and $\nu_{\mathrm{IF}}=6 \mathrm{GHz}$. The data were taken during the performance verification (PV) phase using the double beam switch observing mode with a throw of 2.5 to the SW. The position observed is RA 20:39:02.38, Dec +42:19:33.5 (J2000), close to radio peak C from Roelfsema et al. (1989). A strip map was made in the N-S direction, spanning offsets from $+90^{\prime \prime}$ to $-90^{\prime \prime}$ at a $100^{\prime} 5$ spacing, half the beam size of $21^{\prime \prime}$ FWHM at our observing frequency, which corresponds to $0.17 \mathrm{pc}$ at the distance of DR21. This beam size was measured before launch and is $10 \%$ larger than the diffraction limit due to spillover effects.

Data were taken with two backends: the acousto-optical wide-band spectrometer (WBS) which covers $1140 \mathrm{MHz}$ bandwidth at $1.1 \mathrm{MHz}\left(0.30 \mathrm{~km} \mathrm{~s}^{-1}\right)$ resolution, and the correlator-based high-resolution spectrometer (HRS), which covers $230 \mathrm{MHz}$ bandwidth at $0.48 \mathrm{MHz}\left(0.13 \mathrm{~km} \mathrm{~s}^{-1}\right)$ resolution. Two polarizations are available except for the HRS data of $\mathrm{H}_{2} \mathrm{O}$.

The system temperature of our data is $340-360 \mathrm{~K}$ DSB and the integration time is $67 \mathrm{~s}$ per position $(\mathrm{ON}+\mathrm{OFF})$. Calibration of the raw data onto $T_{\mathrm{A}}^{*}$ scale was performed by the in-orbit system (Roelfsema et al., in prep.); conversion to $T_{\mathrm{mb}}$ was done assuming a beam efficiency of 0.67 as estimated by the Ruze formula and validated by raster maps of Saturn (M. Olberg, priv. comm.). Currently, the flux scale is accurate to $\approx 10 \%$ which will improve when the telescope efficiency and sideband ratio are measured on Mars. The calibration of the data was performed in the Herschel interactive processing environment (HIPE; Ott 2010) version 2.1; further analysis was done within the CLASS ${ }^{1}$. After inspection, data from the two polarizations were averaged together to obtain rms noise levels of $97 \mathrm{mK}$ on $0.5 \mathrm{MHz}$

\footnotetext{
${ }^{1}$ http://WWW.iram.fr/IRAMFR/GILDAS package
}

channels for the WBS data, $195 \mathrm{mK}$ on $0.24 \mathrm{MHz}$ channels for the ${ }^{13} \mathrm{CO}$ HRS data and $244 \mathrm{mK}$ on $0.24 \mathrm{MHz}$ channels for the $\mathrm{H}_{2} \mathrm{O}$ HRS data.

\section{Results}

Figure 1 shows the WBS spectra from both receiver sidebands at each of the 18 offset positions. The spectra from both backends show a continuum signal and two line features. The first line feature is seen in emission at $v=1101.357 \mathrm{GHz}$ LSB or $1114.623 \mathrm{GHz}$ USB, which we identify with the ${ }^{13} \mathrm{CO} J=10 \rightarrow 9$ line at $1101.349597 \mathrm{GHz}^{2}$. This line has an upper level energy $\left(E_{\mathrm{u}}\right)$ of $291 \mathrm{~K}$ and a critical density of $1 \times 10^{6} \mathrm{~cm}^{-3}$, using collision data from Flower (2001). The second feature has a mixed emission-absorption profile and lies at $v=1102.423 \mathrm{GHz}$ LSB or $1113.557 \mathrm{GHz}$ USB, which we identify with the $\mathrm{H}_{2} \mathrm{O} J_{\mathrm{K}_{\mathrm{p}} \mathrm{K}_{\mathrm{o}}}=1_{11} \rightarrow 0_{00}$ line at $1113.34306 \mathrm{GHz}$. This line has $E_{\mathrm{u}}=53 \mathrm{~K}$ and a critical density of $3-6 \times 10^{8} \mathrm{~cm}^{-3}$, using collision data from Faure et al. (2007). No other lines are detected.

Figure 2 shows the HRS spectra of the ${ }^{13} \mathrm{CO}$ and $\mathrm{H}_{2} \mathrm{O}$ lines at the central position, with the continuum subtracted. The ${ }^{13} \mathrm{CO}$ line profile has a double structure which is seen to be well reproduced by the sum of two Gaussians: a narrow component $\left(\Delta V=4.9 \mathrm{~km} \mathrm{~s}^{-1}\right)$ centered at $V_{\mathrm{LSR}}=-3.2 \mathrm{~km} \mathrm{~s}^{-1}$, which we attribute to the molecular cloud core (protostellar envelope), and a broad component $\left(\Delta V=15.2 \mathrm{~km} \mathrm{~s}^{-1}\right)$ centered at $V_{\mathrm{LSR}}=-2.0 \mathrm{~km} \mathrm{~s}^{-1}$, which we ascribe to the molecular outflow. In contrast, the $\mathrm{H}_{2} \mathrm{O}$ line profile shows two absorption features, with strong emission in between, and weaker emission on the far blue- and redshifted sides. Overplotted is a decomposition with three Gaussian components: broad $(\Delta V=$ $23.8 \mathrm{~km} \mathrm{~s}^{-1}$ ) emission centered at $V_{\mathrm{LSR}}=-1.0 \mathrm{~km} \mathrm{~s}^{-1}$, and absorptions at $V_{\mathrm{LSR}}=-2.6 \mathrm{~km} \mathrm{~s}^{-1}$ and $+7.6 \mathrm{~km} \mathrm{~s}^{-1}$ with $\Delta V=5.0$ and $3.0 \mathrm{~km} \mathrm{~s}^{-1}$. This shape resembles the prediction by Poelman \& van der Tak (2007) for the $\mathrm{H}_{2} \mathrm{O}$ ground state lines towards

\footnotetext{
${ }^{2}$ Spectroscopic data are taken from the CDMS catalog (Müller et al. 2005) at http://cdms. de
} 

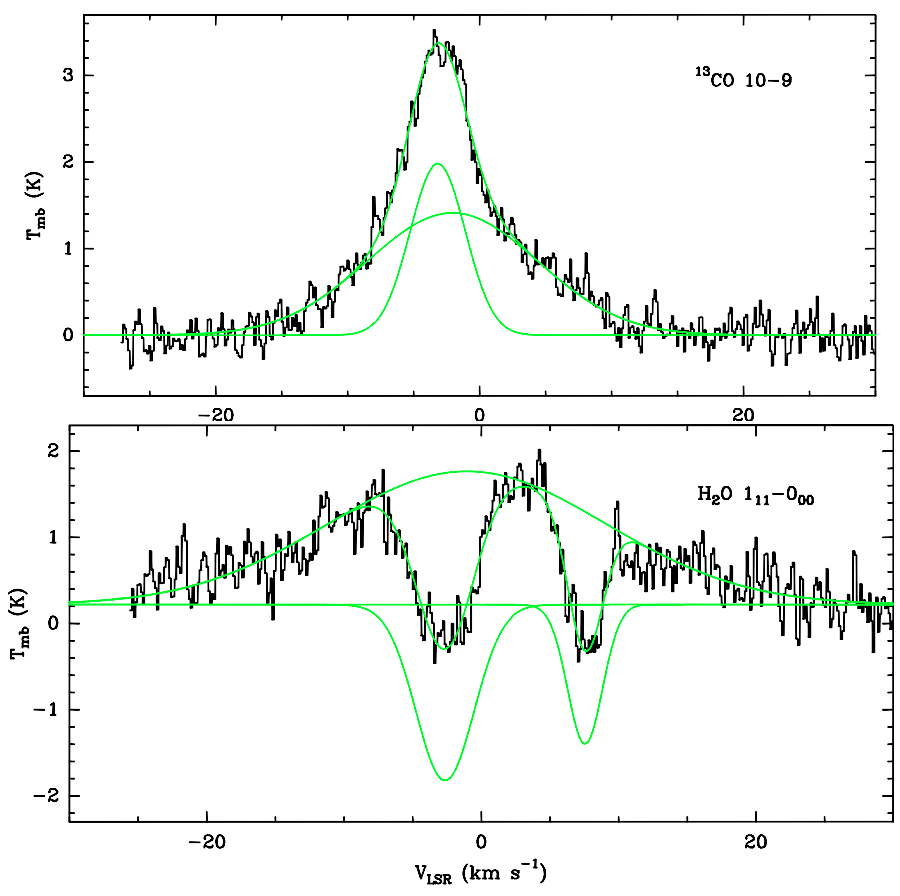

Fig. 2. Spectra of ${ }^{13} \mathrm{CO} 10-9$ (top) and $\mathrm{H}_{2} \mathrm{O} 1_{11}-0_{00}$ (bottom) lines toward the central position, taken with the HRS backend, with Gaussian decompositions overplotted.

high-mass protostellar envelopes for the case of a constant, low $\mathrm{H}_{2} \mathrm{O}$ abundance $\left(\mathrm{H}_{2} \mathrm{O} / \mathrm{H}_{2} \sim 10^{-9}\right)$ with two modifications. First is the broad emission also seen in ${ }^{13} \mathrm{CO}$ which is likely due to the outflow. Second is the absorption at $V_{\mathrm{LSR}}=7.6 \mathrm{~km} \mathrm{~s}^{-1}$ which is known from ground-based observations of low- $J$ CO lines (Jakob et al. 2007) and likely due to a foreground cloud. This absorption is not seen in the ${ }^{13} \mathrm{CO} 10-9$ line, nor in ground-based observations of mid- $J \mathrm{HCN}$ and $\mathrm{HCO}^{+}$lines, which indicates a low temperature $(\sim 10 \mathrm{~K})$ and density $\left(\$ 10^{4} \mathrm{~cm}^{-3}\right)$ for the foreground cloud. Both absorptions are also seen in the SWAS spectra of the $\mathrm{o}-\mathrm{H}_{2} \mathrm{O}$ and $\mathrm{C}^{0}$ ground-state lines, but not in ${ }^{13} \mathrm{CO} 5-4$ (Ashby et al. 2000).

Figure 3 shows the spatial brightness distribution of the continuum and the lines. The points are the results of Gaussian profile fits for the lines, and of linear baseline fits for the continuum, at each offset position. For the $\mathrm{H}_{2} \mathrm{O}$ line, separate fits were made for the emission and the two absorption components. The curves in Fig. 3 are Gaussian fits to the observed spatial distribution. The dust emission peaks at an offset of $(8.7 \pm 0.5)^{\prime \prime}$ North of the nominal position, and the emission distribution has an FWHM width of $(35.7 \pm 0.5)^{\prime \prime}$, consistent with groundbased measurements at longer wavelengths (Gibb et al. 2005; Motte et al. 2007). The peak of the ${ }^{13} \mathrm{CO}$ emission is at offset $(9.65 \pm 0.5)^{\prime \prime}$, consistent with the dust peak within the combined error, but the FWHM of the ${ }^{13} \mathrm{CO}$ emission is $(24.1 \pm 0.4)^{\prime \prime}$, essentially unresolved. The $\mathrm{H}_{2} \mathrm{O}$ emission component has an FWHM of $(25.2 \pm 2.5)^{\prime \prime}$, similar to ${ }^{13} \mathrm{CO}$, but its peak is shifted further South, at offset $(6.4 \pm 1.1)^{\prime \prime}$. The spatial distribution of the $\mathrm{H}_{2} \mathrm{O}$ absorption at $V=-3 \mathrm{~km} \mathrm{~s}^{-1}$ is the same as that of the $\mathrm{H}_{2} \mathrm{O}$ emission, peaking at offset $(6.7 \pm 1.6)^{\prime \prime}$ with an FWHM of $(26.2 \pm 3.3)^{\prime \prime}$. In contrast, the $\mathrm{H}_{2} \mathrm{O}$ absorption at $V=+7.5 \mathrm{~km} \mathrm{~s}^{-1}$ peaks much further North, at offset $(14.2 \pm 1.9)^{\prime \prime}$, and is possibly extended with an FWHM of $(32.8 \pm 4.7)^{\prime \prime}$, which is a lower limit to its full width because of insufficient background signal. Indeed, Schneider et al. (2006) find that the W75N cloud extends over several arcminutes.
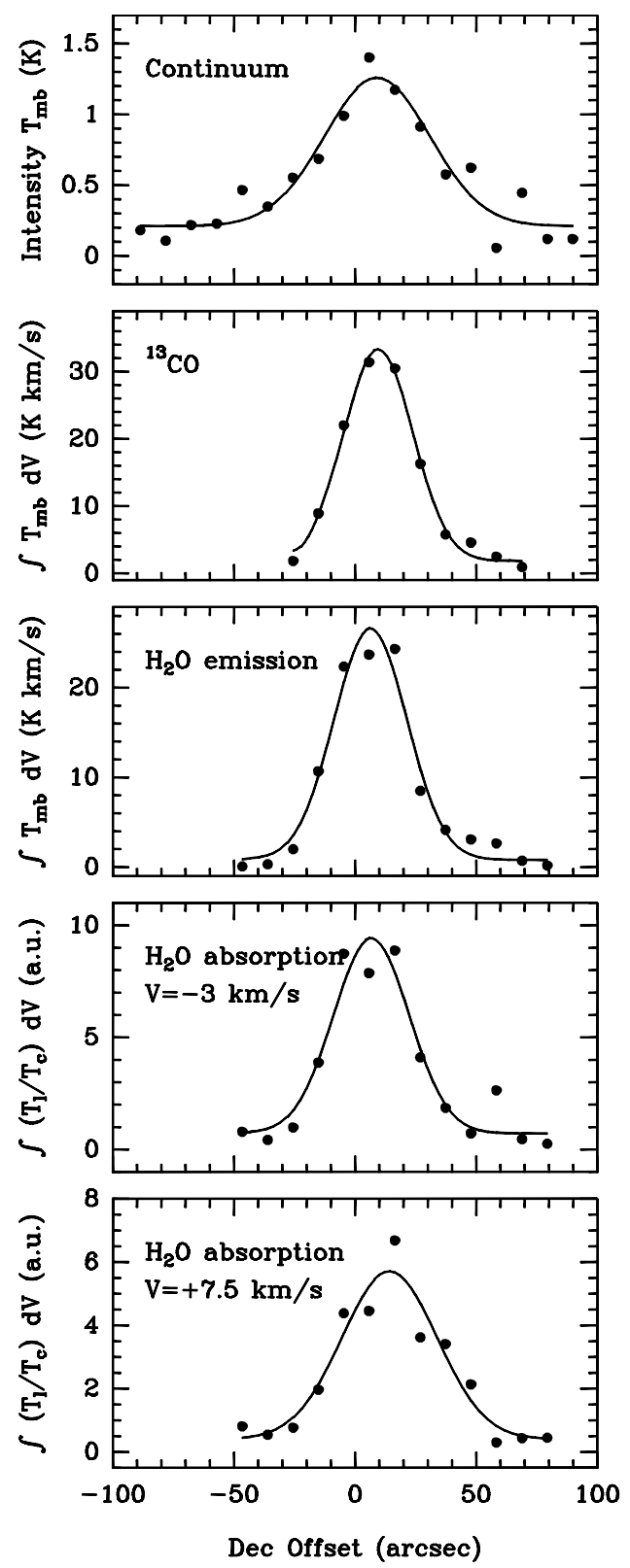

Fig. 3. Plots of observed intensity versus spatial offset with Gaussian models superposed.

\section{Discussion and conclusions}

To estimate the $\mathrm{H}_{2} \mathrm{O}$ and ${ }^{13} \mathrm{CO}$ abundances from our data, we have run spherical radiative transfer models following Marseille et al. (2008). First, the dust continuum emission was modeled with the MC3D program (Wolf \& Henning 2000) with the source size and luminosity kept fixed at the values in Sect. 1. The continuum data are consistent with a power-law density profile $n=n_{0}\left(r / r_{0}\right)^{-\alpha}$ with the index $\alpha=1.5$ as expected for evolved protostellar envelopes (Van der Tak et al. 2000). Derived temperatures range from $117 \mathrm{~K}$ at the adopted inner radius of $0.01 \mathrm{pc}$ to $23 \mathrm{~K}$ at the outer radius of $0.3 \mathrm{pc}$; densities drop from $3 \times 10^{7} \mathrm{~cm}^{-3}$ to $2 \times 10^{5} \mathrm{~cm}^{-3}$. This temperature and density profile was adopted for the line radiative transfer with the RATRAN program (Hogerheijde \& van der Tak 2000). The abundance of $\mathrm{H}_{2} \mathrm{O}$ was varied between $10^{-10}$ and $10^{-7}$ and the ${ }^{13} \mathrm{CO}$ abundance between $10^{-7}$ and $10^{-6}$, both independent of radius.

The red lines in Fig. 4 show the results of our best-fit model. The fit to the line profiles at the central few positions is good if 

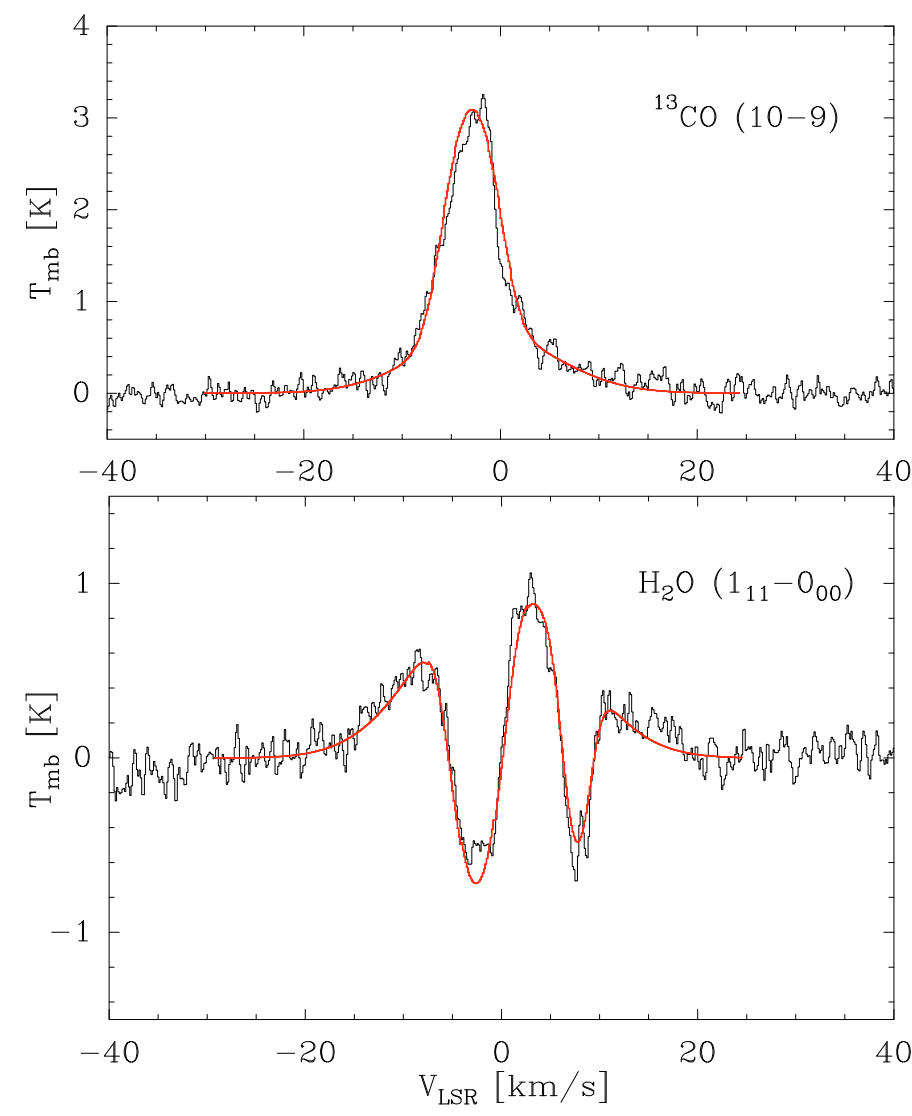

Fig. 4. Data from Fig. 2 with radiative transfer models superposed.

Table 1. Abundances and column densities of ${ }^{13} \mathrm{CO}$ and $\mathrm{H}_{2} \mathrm{O}$.

\begin{tabular}{ccccc}
\hline \hline Component & $\begin{array}{c}N\left({ }^{13} \mathrm{CO}\right)^{a} \\
10^{16} \mathrm{~cm}^{-2}\end{array}$ & $\begin{array}{c}N\left(\mathrm{p}-\mathrm{H}_{2} \mathrm{O}\right) \\
10^{12} \mathrm{~cm}^{-2}\end{array}$ & $\begin{array}{c}N\left(\mathrm{H}_{2}\right) \\
10^{21} \mathrm{~cm}^{-2}\end{array}$ & $x\left(\mathrm{p}-\mathrm{H}_{2} \mathrm{O}\right)$ \\
\hline Dense core & $7.8 \times 10^{-7}$ & $\ldots$ & $\ldots$ & $1.6 \times 10^{-10}$ \\
Outflow & 5 & 10000 & 15 & $7 \times 10^{-7}$ \\
Foreground & 0.7 & 4 & 2.1 & $4 \times 10^{-9}$ \\
\hline
\end{tabular}

Notes. ${ }^{(a)}$ Abundance for the dense core.

two components are added to the dense core model: one representing the outflow and one for the foreground cloud. This simple addition procedure is valid because the foreground cloud is transparent and the outflow is seen at an angle, so that neither component blocks the view of the dense core. The modeled optical depths are 2.8 for the $\mathrm{H}_{2} \mathrm{O}$ line and 0.77 for ${ }^{13} \mathrm{CO}$, and the derived abundances are uncertain to $\approx 35 \%$ which is the quadratic sum of $30 \%$ from the line intensity and $20 \%$ from the core mass estimate. The model underproduces the observed line strengths at large position offsets, which suggests that the density profile flattens out at large radii, as also indicated by the observed continuum brightness at the offset positions.

Deriving the ${ }^{13} \mathrm{CO}$ and $\mathrm{H}_{2} \mathrm{O}$ abundances in the outflow and the foreground using radiative transfer models is not possible because the masses and $\mathrm{H}_{2}$ column densities of these components are unknown. Instead we have used RADEX (Van der Tak et al. 2007) to estimate their ${ }^{13} \mathrm{CO}$ and $\mathrm{H}_{2} \mathrm{O}$ column densities, and use a ${ }^{12} \mathrm{C} /{ }^{13} \mathrm{C}$ ratio of 60 (Milam et al. 2005) and a $\mathrm{CO}$ abundance of $2 \times 10^{-4}$ (Lacy et al. 1994) to convert $N\left({ }^{13} \mathrm{CO}\right)$ to $N\left(\mathrm{H}_{2}\right)$ and estimate $x\left(\mathrm{H}_{2} \mathrm{O}\right)$ as $N\left(\mathrm{H}_{2} \mathrm{O}\right) / N\left(\mathrm{H}_{2}\right)$. For the foreground cloud, we adopt $T_{\text {kin }}=10 \mathrm{~K}$ and $n\left(\mathrm{H}_{2}\right)=10^{4} \mathrm{~cm}^{-3}$, and for the outflow, we adopt $T_{\text {kin }}=200 \mathrm{~K}$ and $n\left(\mathrm{H}_{2}\right)=3 \times 10^{4} \mathrm{~cm}^{-3}$, which assumptions introduce a factor of $\sim 2$ uncertainty each. To estimate $N\left({ }^{13} \mathrm{CO}\right)$ for the foreground cloud, we use the $J=1-0$ observations by Jakob et al. (2007).

Table 1 summarizes our derived column densities and abundances of ${ }^{13} \mathrm{CO}$ and $\mathrm{H}_{2} \mathrm{O}$ in the various physical components of the DR21 region. Our $\mathrm{H}_{2} \mathrm{O}$ abundance in the dense core is $\sim 100 \times$ lower than previous determinations (Sect. 1) but should be regarded as a lower limit. At the low temperatures and high densities in the core, most $\mathrm{H}_{2} \mathrm{O}$ is likely frozen on grains, and the observed line may arise in a small region with a high $\mathrm{H}_{2} \mathrm{O}$ abundance. The derived ${ }^{13} \mathrm{CO}$ abundance for the core is $\sim 4 \times$ lower than expected for the above values of the $\mathrm{CO}$ isotopic ratios and abundance, which suggests that even some $\mathrm{CO}$ is frozen out in the outer parts of the core. The density of the foreground cloud is too low for significant freeze-out, but with $A_{\mathrm{V}} \approx 1.2 \mathrm{mag}$, photodissociation is rapid for $\mathrm{H}_{2} \mathrm{O}$ but not for ${ }^{13} \mathrm{CO}$.

The high $\mathrm{H}_{2} \mathrm{O}$ abundance for the outflow is likely related to its temperature of $\sim 200 \mathrm{~K}$, which is high enough to have $\mathrm{H}_{2} \mathrm{O}$ released from the dust grains by thermal evaporation, or possibly by shocks (Melnick et al. 2008). Further enhancement may be expected in even warmer gas $(\gtrsim 250 \mathrm{~K})$ when neutral-neutral reactions drive most gas-phase oxygen into $\mathrm{H}_{2} \mathrm{O}$, but such gas is not probed by our data. Future HIFI observations of highexcitation $\mathrm{H}_{2} \mathrm{O}$ lines towards protostars of all masses will however very likely reveal this effect.

Acknowledgements. HIFI has been designed and built by a consortium of institutes and university departments from across Europe, Canada and the US under the leadership of SRON Netherlands Institute for Space Research, Groningen, The Netherlands with major contributions from Germany, France and the US. Consortium members are: Canada: CSA, U. Waterloo; France: CESR, LAB, LERMA, IRAM; Germany: KOSMA, MPIfR, MPS; Ireland, NUI Maynooth; Italy: ASI, IFSI-INAF, Arcetri-INAF; Netherlands: SRON, TUD; Poland: CAMK, CBK; Spain: Observatorio Astronómico Nacional (IGN), Centro de Astrobiología (CSIC-INTA); Sweden: Chalmers University of Technology MC2, RSS \& GARD, Onsala Space Observatory, Swedish National Space Board, Stockholm University - Stockholm Observatory; Switzerland: ETH Zürich, FHNW; USA: Caltech, JPL, NHSC.

\section{References}

Ashby, M. L. N., Bergin, E. A., Plume, R., et al. 2000, ApJ, 539, L115 Bjerkeli, P., Liseau, R., Olberg, M., et al. 2009, A\&A, 507, 1455 Braz, M. A., \& Epchtein, N. 1983, A\&AS, 54, 167 de Graauw, Th., et al. 2010, A\&A, 518, L6

Faure, A., Crimier, N., Ceccarelli, C., et al. 2007, A\&A, 472, 1029 Flower, D. R. 2001, J. Phys. B At. Mol. Phys., 34, 2731

Garden, R., Hayashi, M., Hasegawa, T., et al. 1991, ApJ, 374, 540

Gibb, A. G., Thompson, M. A., Wyrowski, F., \& Hatchell, J. 2005, P\&P V, 8194 Hogerheijde, M. R., \& van der Tak, F. F. S. 2000, A\&A, 362, 697 Jakob, H., Kramer, C., Simon, R., et al. 2007, A\&A, 461, 999 Kirby, L. 2009, ApJ, 694, 1056

Lacy, J. H., Knacke, R., Geballe, T. R., \& Tokunaga, A. T. 1994, ApJ, 428, L69 Marseille, M., Bontemps, S., Herpin, F., et al. 2008, A\&A, 488, 579 Melnick, G. J., \& Bergin, E. A. 2005, Adv. Space Res., 36, 1027 Melnick, G. J., Tolls, V., Neufeld, D. A., et al. 2008, ApJ, 683, 876 Milam, S., Savage, C., Brewster, M., et al. 2005, ApJ, 634, 1126 Motte, F., Bontemps, S., Schilke, P., et al. 2007, A\&A, 476, 1243 Müller, H., Schlöder, F., Stutzki, J., et al. 2005, J. Mol. Struct., 742, 215 Ott, J. 2010, ADASS XIX, in press

Pilbratt, G. L., et al. 2010, A\&A, 518, L1

Poelman, D. R., \& van der Tak, F. F. S. 2007, A\&A, 475, 949

Roelfsema, P. R., Goss, W. M., \& Geballe, T. R. 1989, A\&A, 222, 247

Schneider, N., Bontemps, S., Simon, R., et al. 2006, A\&A, 458, 855

Van der Tak, F., van Dishoeck, E., Evans, II, N., et al. 2000, ApJ, 537, 283

Van der Tak, F., Walmsley, C., Herpin, F., et al. 2006, A\&A, 447, 1011

Van der Tak, F., Black, J., Schöier, F., et al. 2007, A\&A, 468, 627

Van Dishoeck, E. F., \& Helmich, F. P. 1996, A\&A, 315, L177

Watson, D. M., Bohac, C. J., Hull, C., et al. 2007, Nature, 448, 1026

Wolf, S., \& Henning, T. 2000, Comp. Phys. Comm., 132, 166

Zinnecker, H., \& Yorke, H. W. 2007, ARA\&A, 45, 481 
1 SRON Netherlands Institute for Space Research, Landleven 12, 9747 AD Groningen, The Netherlands

e-mail: vdtak@sron.nl

2 Kapteyn Institute, University of Groningen, The Netherlands

3 Laboratoire d'Astrophysique de Bordeaux, Floirac, France

${ }^{4}$ Max-Planck-Institut für Radioastronomie, Bonn, Germany

5 Sterrewacht, Universiteit Leiden, The Netherlands

${ }^{6}$ KOSMA, I. Physik. Institut, Universität zu Köln, Germany

7 CESR, Université de Toulouse, France

8 Chalmers University of Technology, 41296 Göteborg, Sweden

9 Institute of Astronomy, ETH Zürich, 8093 Zürich, Switzerland

10 Joint ALMA Observatory, Santiago, Chile

11 European Space Astronomy Centre, ESA, Madrid, Spain

12 Harvard-Smithsonian Center for Astrophysics, Cambridge, USA

13 Denison University, Granville OH, USA

14 MPI für Extraterrestrische Physik, Garching, Germany
15 School of Physics and Astronomy, University of Leeds, UK

${ }^{16}$ Herzberg Institute of Astrophysics, Victoria, Canada

17 Dept. of Physics and Astronomy, University of Victoria, Canada

18 Observatorio Astronómico Nacional, Alcalá de Henares, Spain

19 INAF - Istituto di Fisica dello Spazio Interplanetario, Roma, Italy

20 Dept. of Astronomy, University of Michigan, Ann Arbor, USA

21 California Institute of Technology, Pasadena CA 91125, USA

22 CAB, INTA-CSIC, Torrejón de Ardoz, Spain

${ }^{23}$ University of Amsterdam, The Netherlands

${ }^{24}$ LERMA and UMR 8112 du CNRS, Observatoire de Paris, France

25 Dept. of Physics and Astronomy, University of Waterloo, Canada

${ }^{26}$ Centre for Star and Planet Formation, U. of Copenhagen, Denmark

27 Department of Astronomy, Stockholm University, Sweden

28 Johns Hopkins University, Baltimore, USA

29 JPL, California Institute of Technology, Pasadena, CA 91109, USA

30 Dept. of Physics and Astronomy, University of Calgary, Canada

31 Observatoire de Paris-Meudon, Meudon, France 